\title{
Philosophiques
}

\section{Émotions fortes et constructionnisme faible}

\section{Luc Faucher}

Volume 26, numéro 1, printemps 1999

URI : https://id.erudit.org/iderudit/004921ar

DOI : https://doi.org/10.7202/004921ar

Aller au sommaire du numéro

\section{Éditeur(s)}

Société de philosophie du Québec

\section{ISSN}

0316-2923 (imprimé)

1492-1391 (numérique)

Découvrir la revue

\section{Citer cet article}

Faucher, L. (1999). Émotions fortes et constructionnisme faible. Philosophiques, 26(1), 1-33. https://doi.org/10.7202/004921ar

\section{Résumé de l'article}

De l'avis de plusieurs, il existe une tension entre le modèle " naturaliste » des émotions qui inspire les psychologues évolutionnistes et le modèle des constructionnistes sociaux. D'un côté, il semble que les émotions sont innées, rigides et panculturelles; de l'autre, elles sont conçues comme des artefacts culturels, changeant au gré des arrière-plans culturels. Nous examinons chacune des deux approches et montrons que l'approche naturaliste n'est pas incompatible avec une forme de constructionnisme faible, en ce que la première peut tenir compte d'un certain degré de plasticité. Nous soutenons qu'en fait les deux courants devraient être vus comme offrant des points de vue différents, mais complémentaires, sur les mêmes phénomènes. Cependant, une part du domaine des émotions étudiée par les constructionnistes échappe aux efforts d'intégration. Une façon de remédier à la situation est de proposer un réaménagement conceptuel du domaine. Par exemple, Griffiths $(1990,1997)$ propose que la solution passe par l'élimination du concept d'“ émotion ». Quoique notre investigation des différentes formes d'émotions suggère un certain scepticisme concernant l'utilité théorique du concept d'« émotion ", nous croyons qu'il est trop tôt pour en annoncer la mort.
Tous droits réservés (C) Société de philosophie du Québec, 1999
Ce document est protégé par la loi sur le droit d'auteur. L'utilisation des services d'Érudit (y compris la reproduction) est assujettie à sa politique d'utilisation que vous pouvez consulter en ligne.

https://apropos.erudit.org/fr/usagers/politique-dutilisation/ 


\title{
Émotions fortes et constructionnisme faible
}

\author{
LUC FAUCHER \\ Dept of philosophy \\ Rutgers University \\ lucfaucher@hotmail.com
}

\begin{abstract}
RÉSUMÉ. - De l'avis de plusieurs, il existe une tension entre le modèle «naturaliste » des émotions qui inspire les psychologues évolutionnistes et le modèle des constructionnistes sociaux. D'un côté, il semble que les émotions sont innées, rigides et panculturelles; de l'autre, elles sont conçues comme des artefacts culturels, changeant au gré des arrière-plans culturels. Nous examinons chacune des deux approches et montrons que l'approche naturaliste n'est pas incompatible avec une forme de constructionnisme faible, en ce que la première peut tenir compte d'un certain degré de plasticité. Nous soutenons qu'en fait les deux courants devraient être vus comme offrant des points de vue différents, mais complémentaires, sur les mêmes phénomènes. Cependant, une part du domaine des émotions étudiée par les constructionnistes échappe aux efforts d'intégration. Une façon de remédier à la situation est de proposer un réaménagement conceptuel du domaine. Par exemple, Griffiths (1990, 1997) propose que la solution passe par l'élimination du concept d' «émotion ». Quoique notre investigation des différentes formes d'émotions suggère un certain scepticisme concernant l'utilité théorique du concept d'« émotion », nous croyons qu'il est trop tôt pour en annoncer la mort.
\end{abstract}

\begin{abstract}
According to many, there is an incongruity between the naturalist's model of emotions that inspired the evolutionary psychologists and the social constructionist's model. In brief, the naturalists propose that emotions are innate, rigid and pancultural ; whereas the social constructionists believe that emotions are cultural artefacts without any biological reality. We examine each approach and show that the naturalists' view is not incompatible with a weak form of constructionism. We argue that in fact the two positions should be considered as offering different but complementary views of the same phenomena. However, a part of the domain of emotions studied by the social constructionists escapes the attempt at integration. One way to solve this problem is to reconfigure the domain. Griffith $(1990,1997)$ proposed to do so by eliminating the concept of « emotion ». Our investigation of different forms of emotions suggests that if scepticism concerning the theoretical utility of the concept of «emotion » is grounded, it is still too early to proclaim its death.
\end{abstract}

Les recherches sur les émotions figurent, selon plusieurs, parmi les meilleurs travaux d'un nouveau programme de recherche qui semble vouloir prendre de plus en plus d'importance en psychologie : la psychologie évolutionniste. Comme son nom l'indique, ce programme vise à établir des rapports dynamiques entre la psychologie (principalement les sciences cognitives) et la biologie évolutionniste dans l'espoir que la compréhension des processus qui ont façonné l'esprit humain pourra nous éclairer en retour sur son architecture (et vice versa). Une des thèses de ce programme est que l'esprit est constitué de 
nombreux organes mentaux — « modules» ou "algorithmes darwiniens " - sélectionnés afin de répondre à des problèmes adaptatifs particuliers posés par l'environnement dans lequel nous avons évolué. La supposition d'une modularité massive distingue la psychologie évolutionniste (PE) du Modèle Standard en Sciences Sociales (MSSS), selon lequel l'esprit humain est constitué d'un nombre restreint de processus généraux permettant de résoudre indifféremment l'ensemble de ces problèmes. Ce dernier modèle, accepté par une majorité de sociologues, d'anthropologues, de psychologues et de philosophes, est également largement environnementaliste, concevant l'esprit à l'état initial comme une tabula rasa, la cause structurante de l'esprit humain étant la culture ou l'environnement social.

Reflet de cette divergence fondamentale au sujet de l'esprit, le débat contemporain sur les émotions a eu tendance à se polariser autour de deux grandes positions : le naturalisme et le constructionnisme. Les tenants du naturalisme (pour la plupart des psychologues et des neurologues) mettent l'accent sur le caractère panculturel, non acquis des émotions, ainsi que sur leur continuité phylogénétique ; les tenants du constructionnisme (pour la plupart des chercheurs en sciences sociales) insistent plutôt sur les différences entre les cultures, entre les langages, sur le rôle de chaque société dans la constitution des émotions. Plusieurs observateurs (Lewis et Saarni 1987; Lutz et White 1986) perçoivent une tension entre les deux approches et les considèrent comme irréconciliables. Je montrerai dans la dernière partie de ce texte que nous avons probablement affaire ici à une fausse dichotomie qui repose, entre autres, sur un modèle erroné du développement. Je soutiendrai qu'une version faible du constructionnisme est compatible avec l'approche naturaliste et qu'elle doit être considérée comme complémentaire à celle-ci. Cette attitude, je crois, est dans l'esprit de la psychologie évolutionniste, dont le but n'est pas la réduction des théories de niveau supérieur aux théories de niveau inférieur, mais plutôt l'intégration verticale des différents domaines d'investigation (voir Faucher 1998, chapitre 2).

Avant de discuter de ce problème, je décrirai les travaux les plus représentatifs et les thèses principales de la psychologie évolutionniste dans le domaine des émotions. J'examinerai ensuite quelques travaux récents au sujet des circuits nerveux spécifiques aux émotions venant appuyer les thèses évolutionnistes. Cet intérêt pour la neurologie distingue l'approche que je propose des travaux plus traditionnels sur la philosophie des émotions, qui utilisent uniquement l'analyse conceptuelle comme méthodologie pour mettre au jour la véritable nature des émotions. Je me range donc plutôt ici aux côtés de Solomon pour affirmer que :

[...] Il est maintenant clair que les philosophes ne peuvent plus ignorer ou négliger la riche littérature neurophysiologique sur les émotions [...]. Les philosophes peuvent continuer à soutenir qu'Aristote savait tout au sujet des émotions même s'il ne connaissait rien [he did not know beans] au sujet du cerveau, mais ils le font à leur péril — et en dépit de ce fait évident que parmi 
les faits qui ont transformé le plus radicalement l'histoire de la philosophie et ses concepts se trouvent les nouvelles avancées dans des sciences auparavant inconnues ou non-développées. (1993, pp. 10-11)

Le recours à ce type d'information au sujet des mécanismes émotionnels nous permettra de capturer une bonne partie de la théorie du sens commun concernant les émotions (par exemple leur caractère involontaire et irrationnel, qui semble être si central, ainsi que leur intentionnalité), tout en la corrigeant sur certains points. Puisqu'une bonne partie de la philosophie des émotions n'a consisté jusqu'à maintenant qu'à articuler et discuter cette théorie implicite, la prise en compte de la littérature neurophysiologique devrait permettre de jeter un éclairage nouveau sur les problèmes séculaires de la philosophie et d'élaborer une théorie plus adéquate empiriquement. J'utiliserai ensuite certaines idées tirées de la réflexion sur les particularités de l'environnement adaptatif des humains pour proposer des hypothèses sur l'existence de certaines émotions spécifiques aux primates supérieurs. J'utiliserai les données provenant des travaux sur la psychopathie pour suggérer certains mécanismes développementaux responsables de l'apparition de ces émotions. Je décrirai finalement les thèses centrales du constructionnisme, puis je tâcherai de voir si, à la lumière des données provenant des neurosciences ainsi que de la psychologie du développement, il n'est pas possible de penser des relations de complémentarité entre les deux approches. Mais d'abord, quelques mots sur les travaux les plus importants de ce qu'en PE on considère être le paradigme des mécanismes cognitifs spécialisés : les émotions.

\section{La psychologie évolutionniste et la fonction des émotions \\ 1.1 Ekman et Öhman : deux exemples de travaux dans le paradigme évolutionniste}

Parmi les nombreux travaux sur les émotions qui s'inscrivent dans le paradigme évolutionniste, je m’intéresserai brièvement aux résultats de deux chercheurs qui nous permettront de mieux comprendre les fonctions possibles des émotions, ceux de Ekman et de Öhman.

À travers un ensemble d'expériences destinées à départager l'hypothèse du modelage culturel de l'hypothèse universaliste de Darwin concernant l'expression des émotions, Ekman (1980b, 1984, 1992a, b, c, 1993 ; Ekman et Friesen 1971) a réussi à montrer qu'il existait un ensemble d'expressions faciales panculturelles liées thématiquement aux situations qui déclenchent les émotions chez les Occidentaux. Il montra également que ces expressions pouvaient parfois être masquées, quoiqu'incomplètement (Ekman parle d'une "fuite» ("leakage») de quelques millisecondes de la véritable émotion avant qu'elle soit recouverte par l'émotion désirée), par ce qu'il nomme des « règles d'expression ». Ces règles, propres à une culture donnée, dictent qui peut avoir quelle émotion, devant qui et quand (par exemple, il est tout à fait convenable de pleurer si on est celle qui vient d'être nommée 
Miss Univers, mais celle qui finit deuxième doit sourire). Ekman découvrit également que les expressions véritables ne peuvent être feintes que par une minorité de personnes. Certains muscles, impliqués dans les émotions véritables, ne sont tout simplement pas sous le contrôle volontaire (par exemple, l'orbicularis oculi). La contraction de ces muscles du visage devient ainsi un prédicteur fiable de la présence ou de l'absence d'une émotion.

Parmi les expériences récentes d'Ekman (Ekman et al. 1983), l'une retiendra notre attention : l'activation de la physiologie de l'émotion par l'exécution volontaire d'actions faciales. Dans le cadre de cette expérience, on indiqua aux sujets les muscles faciaux qu'ils devaient contracter. On voulait ainsi créer sur leur visage une expression qui avait été identifiée comme universelle sans que ceux-ci s'en rendent compte. On s'aperçut que ces actions volontaires généraient des changements involontaires du Système Nerveux Autonome (SNA), ainsi que du Système Nerveux Central (SNC). Ces changements étaient identiques à ceux qui surviennent lors de tâches plus standards qui ont pour but de déclencher des émotions. De plus, lorsque les sujets suivaient les instructions à propos des expressions faciales, ils ne rapportaient pas simplement un changement physiologique, mais ils affirmaient faire l'expérience de l'émotion dont ils reproduisaient l'expression.

Ce vers quoi semblent poindre ces travaux empiriques, et de nombreux autres, est que certaines expressions d'émotions sont contrôlées par un programme relativement rigide et panculturel, que Ekman (1980b) nomme programme d'affects (affects-program). Ekman résume ainsi l'hypothèse :

[...] il existe au niveau du système nerveux un programme qui établit une connexion entre les émotions spécifiques et des mouvements musculaires faciaux donnés. [...] les mouvements musculaires faciaux associés à une émotion particulière sont régis par le programme, tant que des règles d'expression ne créent pas d'interférence, et ils sont universels. ( 1980a, p. 1415 ; je souligne)

Ces programmes (ou modules fodoriens au sens où ils sont impénétrables cognitivement) seraient responsables de l'ensemble des réactions qui caractérisent les émotions : non seulement les expressions faciales, mais également les modifications dans l'expression vocale, les réactions des muscles squelettiques (posture) et les changements du système nerveux autonome (augmentation ou diminution du rythme des battements cardiaques) ${ }^{1}$.

I. L'argument principal en faveur de l'existence des programmes d'affects est constitué par l'inférence à la meilleure explication suivante. Il y a un certain nombre de caractéristiques des réponses émotionnelles qui demandent une explication. Parmi celles-ci, notons le fait qu'elles sont brèves, rapides, complexes, organisées et dans une large mesure involontaires. L'argument en faveur de l'existence de programmes d'affects consiste à soutenir que la meilleure explication de ces caractéristiques est l'existence de circuits ou de programmes nerveux emmagasinant les ensembles de réponses propres à chaque émotion et qui sont activés rapidement de façon coordonnée face à quelques stimuli extérieurs. 
Selon Ekman, ces programmes d'affects sont déclenchés par des mécanismes d'évaluation (appraisal mechanisms) relativement indépendants du système central des désirs et des croyances qui guide normalement l'action rationnelle. Cette indépendance permettrait d'expliquer certaines formes d' "akrasie émotionnelle ", c'est-à-dire les cas où les expériences émotionnelles qui se produisent semblent inadéquates par rapport aux croyances et désirs d'un sujet. À titre d'exemple, la littérature sur le dégoût alimentaire est remplie d'anecdotes sur les gens qui ont développé des aversions alimentaires durables après avoir éprouvé des nausées et des vomissements quelques heures après l'ingestion d'un aliment. Même si parfois ceux-ci en viennent à savoir que leurs maux étaient d'origine virale, ils conservent néanmoins une aversion durable vis-à-vis de cet aliment. Ces "illusions émotionnelles » suggèrent l'existence de processus d'évaluation indépendants des systèmes de fixation des croyances, des modules, de la même façon que les illusions perceptuelles. Ces modules pourraient bien expliquer la difficulté rencontrée depuis l'Antiquité à contrôler les émotions uniquement par le travail de la raison. Cette conception irait également à l'encontre de celle, prévalante en philosophie, que Griffiths (1989b, 1990 ; voir également Clark 1986 et Greenspan 1981) nomme le " cognitivisme philosophique ", c'est-à-dire l'idée selon laquelle les émotions peuvent et doivent être analysées uniquement en termes de croyances et de désirs qu'entretiendrait un sujet ${ }^{2}$. Nous verrons plus bas, à la section 3 , comment cette idée trouve encore des échos dans la littérature constructionniste.

Contrairement aux programmes d'affects, les mécanismes d'évaluation, quoiqu'autonomes, ne semblent cependant pas programmés de façon innée. En effet, il n'existe que très peu de stimuli qui déclenchent une réaction émotionnelle chez le nouveau-né (ces stimuli se résument aux sons très forts, à la perte d'équilibre, à la présentation soudaine d'objets, aux goûts amers et aux expressions faciales des proches). Malgré cela, ces mécanismes ne sont pas totalement plastiques. Öhman (1986) montra qu'il existe une certaine préparation à l'apprentissage (learning preparedness) chez les humains et d'autres mammifères, c'est-à-dire une prédisposition à apprendre rapidement une association entre un type de stimuli et un type de réponse ${ }^{3}$.

La thèse de Öhman porte sur la peur, mais on peut assumer qu'elle est généralisable à l'ensemble des émotions que nous nommerons plus loin les

2. Ce « cognitivisme philosophique" ne doit pas être confondu avec le « cognitivisme psychologique " qui fait appel à des notions de "connaissance tacite " par exemple. Pour les psychologues, "[...] le terme "cognitif" [...] réfère aux explications psychologiques en termes de représentation et de transformation qui peuvent être, ou pas, conscientes. La connaissance tacite est utilisée, par exemple, lorsque le coucou, qui niche en Angleterre, quitte le pays à la fin de l'été pour voler vers l'Afrique sans le bénéfice d'un entraînement parental ou lorsqu'un locuteur humain d'une langue énonce des phrases grammaticales même s'il ne peut pas formuler explicitement la syntaxe de sa langue. » (Oatley et Johnson-Laird 1987, p. 30)

3. Lorsque les associations ne sont apprises ni rapidement ni lentement, on parlera de non-préparation et lorsqu'un organisme ne peut apprendre qu'avec une grande difficulté une association, on parlera de contre-préparation. On utilisera également "saillance " pour parler des stimuli auxquels nous sommes plus sensibles. 
"émotions fondamentales ». Öhman et ses collègues maintiennent qu'il existe des stimuli envers lesquels les humains sont prédisposés à acquérir la peur, typiquement les stimuli qui ont indiqué la présence de danger à travers notre histoire évolutionniste. Leurs expériences (rapportées dans Öhman 1986) ont montré que certaines associations entre les stimuli phobiques classiques (ou les "stimuli pertinents " pour la peur (fear-relevant), comme les serpents ou les araignées) ainsi que les stimuli aversifs (comme les chocs électriques) étaient plus faciles à apprendre et plus résistantes à l'extinction que les associations établies entre les stimuli neutres (comme une fleur) ou arbitraires (comme une forme). Öhman cite également une expérience qui montre qu'il est plus facile d'induire la peur et plus difficile de la faire disparaître pour des stimuli comme les serpents et les araignées que pour les revolvers et les fusils. D'autres expériences, rapportées par Marks et Nesse (1994, p. 255), montrent que les bébés rhésus ne naissent pas avec la peur des serpents, mais qu'une peur durable se développe après quelques observations d'un autre rhésus qui montre des signes de peur devant un serpent. Aucune peur de ce type ne se développe après avoir vu un rhésus réagissant avec frayeur devant une fleur, par exemple. Ces expériences montrent que l'association entre quelques stimuli phobiques et le danger est une association préparée chez les humains et les primates (suivant Segal 1996, on nommera le type de mécanisme en charge de cet apprentissage "module diachronique ». Les travaux d'Öhman suggèrent en effet l'existence d'un ou plusieurs "mécanismes d'acquisition de la peur » du même genre que le "mécanisme d'acquisition du langage " de Chomsky).

Cette façon de se représenter les systèmes de déclenchement est sensée d'un point de vue évolutionniste. D'une part, leur contenu n'étant pas déterminé de façon innée, les mécanismes d'évaluation peuvent permettre des réponses adaptatives aux événements qui ont une signification écologique pour l'organisme. Par exemple, la réponse de peur est une réponse aux situations dangereuses. Mais la catégorie biologique des événements dangereux n'a pas une apparence uniforme à travers les générations. Aussi est-il adaptatif pour les programmes de réponses d'être liés à un mécanisme qui peut interpréter les grandes catégories écologiques, comme le danger, à la lumière des conditions locales et qui peut les assimiler à certaines caractéristiques détectables de l'environnement.

S'il est avantageux d'avoir un système flexible, il existe, d'un autre côté, certaines constantes dans l'environnement évolutionniste auxquelles un système d'apprentissage peut être programmé à être sensible. Prenons le cas du dégoût, qui est une réaction destinée à prévenir l'ingestion de substances nocives. On sait qu'il existe des mécanismes chez les rats qui associent la nausée avec la nourriture ingérée. Il existe donc une forme de préparation chez le rat qui favorise l'établissement d'une association entre la nausée et les stimuli gustatifs plutôt que tout autre stimulus. Cette forme de contrainte sur l'apprentissage, qui restreint le dégoût aux stimuli gustatifs, 
est avantageuse. En effet, un des problèmes majeurs dans l'évaluation des aliments potentiellement nutritifs pour un omnivore est le délai, dû à la digestion, entre l'ingestion et ses conséquences métaboliques. Un processus d'apprentissage exhibant un délai temporel conjoint à l'association sélective des indices alimentaires avec les problèmes viscéraux résout ce problème.

Notons enfin, comme le suggère Griffiths (1990), que l'existence de prédispositions permet de donner une explication évolutionniste de l'apparente irrationalité des émotions ainsi que de la persistance irrationnelle des jugements à propos de la signification émotive de l'environnement. Les réponses émotionnelles, on le sait, sont moins facilement ajustables que les croyances lorsqu'une nouvelle information sur le monde est acquise. La peur du noir ou celle des araignées persiste parfois malgré toute l'information dont on dispose par ailleurs au sujet de leur caractère inoffensif. Comme le savent les thérapeutes, il faut parfois un contre-conditionnement intensif pour faire disparaître ces peurs. Ces faits s'expliquent par la relative indépendance des divers systèmes de croyances des sujets.

\subsection{Deux fonctions pour les émotions}

Le consensus qui se dégage des travaux autour de la fonction générale des émotions dans le paradigme évolutionniste (Johnson-Laird 1988 ; Nesse 1990 ; Plutchick 1984 ; Scherer 1993 ; Tooby 1987 ; Tooby et Cosmides 1990) consiste à les concevoir, non plus comme des agents désorganisateurs du comportement comme ce fut traditionnellement le cas, ni uniquement comme des perceptions du corps comme le faisaient Descartes ou James, mais plutôt comme des modes d'opération spécifiques de l'esprit/cerveau permettant la coordination des changements physiologiques, cognitifs et comportementaux. Les émotions sont conçues dans le cadre de la PE comme des programmes qui modifient le mode sous lequel fonctionnent les nombreux mécanismes qui composent l'esprit, de façon à harmoniser leur fonctionnement afin de résoudre les problèmes adaptatifs posés par les situations rencontrées ${ }^{4}$. C'est entre autres ce que soutient Nesse (voir également Tooby 1987) qui, filant la métaphore du programme, écrit :

À plusieurs égards, les émotions procurent pour l'esprit ce que les programmes procurent pour l'ordinateur. [...] Charger un programme change l'apparence de l'écran, les fonctions de certaines clés, l'utilisation de certains processeurs et l'accès à certaines informations. De la même façon, une émotion peut changer l'expression du visage, la réponse à des stimuli particuliers, la tendance à utiliser un mode ou un autre de pensée et la disponibilité de certains souvenirs. (1990, p. 269)

4. L'harmonisation des différents systèmes se ferait non pas par une forme de communication propositionnelle entre ces systèmes, mais plutôt par une forme de communication non propositionnelle : le signal émotionnel se propagerait à travers eux pour les mettre dans un mode particulier de fonctionnement. En ce sens, le signal émotionnel n'a pas de contenu propositionnel ou de structure syntaxique ; sa fonction n'est pas d'informer, mais de contrôler, d'effectuer une transition rapide dans la hiérarchie des processeurs qui constituent notre esprit (Johnson-Laird et Oatley 1992, p. 207). 
Les émotions constitueraient à cet égard un antidote à l'embarras de richesses cognitives dont jouit l'humain. En effet, nos capacités cognitives nous permettent de considérer, pour chaque situation, une myriade de possibilités d'action. Mais, dans certains cas, cet avantage se transforme en désavantage, spécialement lorsqu'il faut agir rapidement. Dans ces cas, nous sommes en proie à ce que Fodor (1987) nomme le problème d'Hamlet, c'està-dire que nous ne savons pas quand nous devrions cesser de penser et commencer à agir. Comme l'écrit Levenson :

Il y a clairement des moments où l'action est plus appropriée que la délibération, où répondre est plus approprié que considérer, où faire est plus approprié que planifier. Dans des situations où l'hésitation pourrait avoir les plus terribles conséquences, l'émotion fonctionne en mettant de côté le traitement cognitif qui est trop encombrant, trop obsessif, trop indulgent avec lui-même [self-indulgent] et, ultimement, qui a trop de chance d'être inconclusif. (Levenson, 1994, p. 124)

Les émotions sont donc conçues comme sélectionnant un nombre limité de modes d'action dont l'efficacité a été testée par le temps. Elles sont des moyens de faire face rapidement à un problème ou à une situation de façon adaptative en coordonnant et imposant un mode de fonctionnement à des sous-systèmes qui fonctionnent habituellement indépendamment les uns des autres, et ce, sans une longue délibération qui est habituellement le propre de la résolution consciente de problèmes.

Les émotions ont également une autre fonction qui n'est pas moins importante et qui est bien connue des éthologues : elles permettent de communiquer nos sentiments et de contrôler le comportement des autres. Les caractéristiques expressives du visage, de la voix et de la posture sont autant de messages qui renseignent les autres sur la façon dont nous nous sentons ou sur nos réactions vis-à-vis de leurs actions. Elles peuvent donc fournir aux autres un feed-back sur leurs actions qui permet une modification de leur projet d'action d'origine, mais elles les renseignent également sur ce que nous sommes susceptibles de faire prochainement, sur nos dispositions à agir. L'expression des émotions joue donc un rôle important dans la coordination sociale.

Des études récentes (Wellman et al. 1995) montrent que nous sommes sensibles à ce type d'information très tôt dans notre enfance, bien avant que nous soyons sensibles à la possibilité que les autres aient des états mentaux (comme des croyances et des désirs) différents des nôtres ${ }^{5}$. Par exemple, les enfants entre 5 et 7 mois qui entendent une voix expressive vont sélectivement

5. Blair (1996) soutient que les autistes, qui selon la majorité des chercheurs ne disposent pas d'une capacité à attribuer des états intentionnels aux autres, réagissent aux émotions des autres ainsi qu'à leur détresse. Une des raisons possibles de cette capacité est justement que des expressions caractéristiques soient associées aux émotions. Les autistes, comme les primates non-humains, semblent posséder une "théorie béhavioriste de l'esprit " (voir Faucher, manuscrit). 
regarder vers une figure qui exprime la même émotion lorsque placés devant plusieurs choix, montrant ainsi leur habileté à détecter la signification commune des présentations optiques et acoustiques des expressions émotionnelles. En fait, il semble que la reconnaissance des états émotionnels à partir des expressions jouent un rôle primordial chez les enfants en guidant leur apprentissage. En regardant les expressions sur le visage de leurs parents ou en étant attentifs aux différentes modulations de leur voix (Fernald 1992), les enfants peuvent obtenir des renseignements sur le caractère de certaines situations ou de certains stimuli.

L'utilisation des expressions émotionnelles pour la communication demande donc non seulement une habileté à émettre ces expressions, mais également une capacité à recevoir ou à décoder ces informations. Si, comme je viens de le mentionner, les expressions émotionnelles jouent un rôle très tôt dans l'apprentissage, on peut penser que le système de décodage responsable de leur interprétation comporte des éléments innés. En d'autres mots, comme ce mécanisme permet l'acquisition des informations culturelles et l'entrée dans la culture, il doit utiliser des données préculturelles. Un tel mécanisme ne peut donc fonctionner que si l'interprétation de certaines expressions est déjà spécifiée pour l'enfant. Mais ce type de système ne peut avoir été sélectionné que s'il existe une certaine régularité des expressions émotionnelles sur laquelle le mécanisme peut tabler. Ici aussi, donc, il est nécessaire de faire référence à des programmes d'affects stables.

\subsection{Les bases neurologiques des programmes d'affects}

Les recherches sur le substrat neuronal des programmes d'affects indiquent que ceux-ci se situent probablement dans les circuits nerveux du système limbique. Le rôle de ces structures sous-corticales dans les émotions a conduit Maclean (1980) à faire l'hypothèse que celles-ci représentent probablement une façon phylogénétiquement plus primitive que celle du néo-cortex de traiter l'information importante et de diriger nos réponses. Cette hypothèse ne fait pas que postuler l'existence de deux systèmes, mais également leur indépendance possible. Dans ce qui suit, j'aimerais considérer quelques travaux récents en neuropsychologie, qui permettent de comprendre comment cette indépendance est possible et quelles en sont les conséquences.

Selon Ledoux (1994a), une partie des fonctions du traitement émotionnel dépend d'une petite structure enfouie dans le lobe temporal : l'amygdale. Les animaux qui souffrent de lésions à cette structure peuvent répondre normalement aux caractéristiques perceptuelles immédiates ou remémorées des objets, mais pas à la signification émotionnelle de ces mêmes objets. La propension de la peur à mener à des comportements stéréotypés et à des modifications viscérales et squelettiques s'explique par la connexion qui existe entre les structures amygdaliennes et les systèmes du tronc cérébral qui sont impliqués dans le contrôle des comportements préprogrammés et spécifiques à l'espèce ainsi qu'entre les systèmes endocriniens et le système nerveux autonome. Par exemple, le noyau central de l'amygdale a accès aux 
noyaux du tronc cérébral qui contrôlent les mouvements faciaux. Cette connexion distingue le système émotionnel des systèmes cognitifs de haut niveau qui sont plutôt connectés avec les aires motrices du cortex responsables du comportement volontaire.

Les inputs du noyau central proviennent du noyau latéral de l'amygdale (NLA). Ce dernier peut recevoir ses informations de deux sources : soit des aires thalamiques, soit des différentes aires associatives du cortex. Les circuits qui commencent dans le thalamus ne fournissent qu'une perception grossière du monde, mais ils ont l'avantage d'être rapides puisqu'ils ne requièrent qu'un seul relais neuronal. Du point de vue neuroanatomique, les prérequis pour l'activation des réactions émotionnelles apparaissent donc minimaux et surtout indépendants du type de transformations qui caractérise habituellement les opérations cognitives supérieures. Les programmes d'affects peuvent être déclenchés directement par les inputs subcorticaux, c'est-à-dire avant que l'intégration perceptuelle n'ait eu lieu et que le système puisse se représenter complètement le stimulus (voir Ledoux, 1994b, p. 57). Il faut croire dans ce cas que le système peut s'activer à partir d'une information incomplète et fragmentaire. Comme l'écrit Ledoux :

[...] la vision d'une forme mince et sinuante, sur le chemin devant nous, suffit à déclencher des réactions défensives. Nous réagissons ainsi avant de nous être assurés que nous avons rencontré un serpent, et nous ne cherchons pas à savoir que les serpents sont des reptiles ou que leur peau peut servir à la fabrication de sacs et de ceintures : ces informations inutiles retarderaient une réaction efficace et rapide, susceptible de nous sauver la vie.

Le cerveau a simplement besoin de stocker les indices élémentaires et de les détecter. Dans un deuxième temps seulement, le traitement des informations de base par le cortex permet une vérification [...] ou la neutralisation de la réaction de peur. (1994b, p. 57)

On le sait, les aires sensorielles du thalamus sont également les portes d'entrée du néo-cortex de l'hippocampe où des représentations distales seront élaborées à partir des signaux sensoriels. Dépendant des aires, ces stimuli seront transformés en représentations d'objets, de concepts ou de contextes qui pourront toutes déclencher, via les structures amygdaliennes, un syndrome émotionnel. On peut donc comprendre que plus les capacités de traitement de ces aires augmentent, plus ce qui déclenchera une émotion pourra être varié et abstrait.

Cette distinction entre les provenances des inputs déclenchants les programmes émotionnels viendrait appuyer les thèses de Zajonc (1980, 1984), qui maintient, contre Lazarus (1984), que les émotions et la cognition sont des systèmes séparés et partiellement indépendants et, par conséquent, que les réactions affectives peuvent survenir sans un encodage perceptif et cognitif élaboré, qu'elles peuvent être, en d'autres mots, précognitives. Bien entendu, la plupart du temps, l'évaluation émotionnelle et l'évaluation 
cognitive fonctionnent de concert, mais elles peuvent parfois fonctionner indépendamment l'une de l'autre. Dans le cas où le système émotionnel fonctionne indépendamment du système cognitif, nous avons affaire à une émotion qui apparaît soit sans raison, soit comme contraire à la raison; dans le cas où l'évaluation est purement cognitive, c'est-à-dire dans le cas où une aire n'enverrait aucun message à la structure amygdalienne, nous ferions face à une émotion "désincarnée ", comme le disait James, c'est-à-dire à une émotion qui ne met pas en marche un programme d'affects.

Finalement, notons qu'il existe d'autres projections qui partent de l'amygdale vers le néo-cortex. Comme le note Ledoux, le champ de projection de celle-ci vers le néo-cortex est plus vaste que le champ des aires du néo-cortex qui projettent vers l'amygdale. Ceci laisse penser que l'amygdale peut influencer plusieurs processus cognitifs. Cette possibilité est amplifiée par le fait que cette structure peut également influencer les processus du tronc cérébral, du cervelet et les systèmes cholinergiques qui jouent un rôle dans l'activation corticale et la vigilance.

À la lumière de ces travaux, on peut mieux comprendre la relative indépendance du système émotionnel vis-à-vis de la cognition, mais également, on peut spéculer sur la nature des interactions entre les deux systèmes. Par exemple, les expériences sur les animaux décérébrés ont montré que ceux-ci continuent à exhiber des comportements émotifs, ce qui est facilement compréhensible si la structure responsable des émotions est l'amygdale. Mais comme le note Panskeep :

[...] privés de leur cortex, les animaux deviennent plus émotifs, ce qui conduit à penser que, pour réaliser ses potentialités cognitives, le cerveau supérieur (ou cortical) a dû inhiber les tendances émotionnelles primitives. $(1989$, p. 61)

Le cortex semble donc jouer un rôle particulier dans la modulation des tendances émotionnelles primitives et est probablement impliqué dans les comportements issus des règles d'expression postulées par Ekman. Qui plus est, il semble que la destruction du cortex n'abolisse pas la mémoire émotionnelle, ce qui indique que celle-ci est probablement distincte de la mémoire déclarative et consciente. Ceci expliquerait la difficulté que nous avons à nous débarrasser des souvenirs émotionnels.

\section{2. Émotions sociales}

Une fois reconnue l'existence de programme d'affects, on peut maintenant se demander combien de ces programmes d'affects existent ? Si on utilise l'étude des substrats neuronaux comme critère, leur nombre semble être de quatre; si on utilise plutôt les expressions faciales, le consensus s'établit autour d'environ cinq ou six : la joie, la tristesse, le dégoût, la peur, la colère et la surprise ${ }^{6}$. Ekman (1994) nomme les émotions qui possèdent un

6. Ekman (1998, p. 390) en propose maintenant huit, ajoutant à la liste ci-haut mentionnée le mépris et la honte. 
programme d'affects " émotions fondamentales ", entendant entre autres par là que ce sont des états qui ont été modelés par la sélection naturelle et que l'on retrouve dans toutes les cultures, de même que dans les espèces infrahumaines qui ont rencontré les mêmes problèmes adaptatifs que nous.

L'étude des expressions émotionnelles a permis à coup sûr d'identifier certaines grandes catégories d'émotions. S'il est vrai que toutes les émotions ayant une expression panculturelle sont fondamentales, on peut cependant se demander si toutes les émotions fondamentales doivent absolument être accompagnées d'une expression faciale particulière. Ekman lui-même concédait récemment (1984, p. 330 et 1994, p. 18) que toutes les émotions n'ont pas nécessairement un signal facial universel. Si les expressions faciales ne sont pas suffisantes pour mener à l'identification des émotions fondamentales, la psychologie évolutionniste n'est pas pour autant complètement démunie. Elle dispose d'un autre moyen de produire des hypothèses sur l'existence de ces émotions. Cette méthode consiste à identifier ce que Lazarus (1991) nomme les "thèmes relationnels" récurrents dans l'histoire adaptative des humains, c'est-à-dire les problèmes adaptatifs qu'ont dû rencontrer et surmonter les humains ${ }^{7}$.

On le sait, le succès reproductif des humains dépend pour une grande part de leur succès social, et le succès social dépend de la négociation et des relations de réciprocité. Ces échanges sociaux sont énormément compliqués par le fait que la tromperie et la défection sont des stratégies toujours ouvertes aux partenaires. Ces conditions spécifiques aux humains (et à quelques primates, voir De Waal 1997) ont posé des problèmes adaptatifs récurrents qui ont demandé des réponses spécifiques (Frank 1988 ; Frank et al. 1993 ; Gibbard 1993 ; Hamburg 1968 ; Nesse 1990). Puisque la coopération atteint chez les humains un niveau inégalé, on peut penser que les problèmes liés à ce type de situations ont été résolus. Les problèmes liés à la transgression des contrats sociaux ou encore ceux concernant la continuation d'une relation entre partenaires sont probablement la cause de l'apparition de toute une classe d'émotions sociales (ou de sentiments moraux) comme la culpabilité, la honte, la jalousie ou l'amour. Ces émotions auraient une double fonction. D'une part, elles agiraient comme motivation permettant au sujet de transcender son intérêt immédiat en faveur de son intérêt matériel ultime ${ }^{8}$. Par exemple, la culpabilité est généralement causée par une situation où l'on a manqué

7. À la fin de cette section, nous tenterons cependant de montrer, en suivant Griffiths (1994, 1997), que l'équivalence fonctionnelle qui est postulée entre les émotions fondamentales dont nous avons parlé au chapitre précédent et les émotions sociales dont nous parlerons est trompeuse. Une explication faisant mention des classifications cladistiques serait probablement plus productive dans le contexte.

8. Ainsi, plutôt que d'être reléguées dans les marges de l'éthique, les émotions pourraient être vues comme y jouant un rôle important. Pour une conception émotiviste de l'éthique, voir Gibbard (1993). 
à ses obligations et elle motive un comportement altruiste excessif vis-à-vis celui à qui nous avons fait défaut, signifiant ainsi notre désir de restaurer sa confiance. Elle peut également empêcher quelqu'un de manquer à ses obligations pour éviter de ressentir ce sentiment désagréable, même si, ce faisant, cette personne va à l'encontre de son intérêt immédiat. Elles résolvent donc ce que Frank (1988) a nommé le "problème de l'engagement "(commitment problem) : par exemple, l'amour et la culpabilité permettent à un couple l'investissement commun de temps et d'énergie pour élever des enfants, ce que des gens poursuivant uniquement leur intérêt immédiat ne pourraient pas faire. D'autre part, les émotions agissent également comme signaux pour les autres, indiquant comment un sujet risque de se comporter dans le futur. La façon dont se comporte le sujet dans certaines situations de coopération, ou les émotions qu'il éprouve dans ces situations, établiront sa "réputation " dans le groupe, à savoir s'il est un " coopérateur " convenable ou un "défecteur " dont il faut se méfier. Selon Frank, pour établir cette réputation, les sujets doivent coopérer même dans des situations où la coopération n'est pas un choix rationnel à court terme (entre autres, dans les situations où la probabilité d'un retour de l'investissement de la part de l'autre est quasi inexistante, comme lorsque vous laissez un pourboire à un serveur dans une ville où vous n'êtes que de passage).

Une façon de vérifier cette hypothèse concernant l'existence d'une classe spécifique d'émotions sociales est d'utiliser la dissociation, une méthode prisée dans l'étude des systèmes complexes et qui permet la décomposition et l'identification des sous-systèmes responsables conjointement d'une capacité particulière. Dans le cas qui nous intéresse, cette méthode consistera à isoler le système (ou un des systèmes) responsable des émotions sociales en utilisant les données fournies par la psychopathologie. Grosso modo, on tentera d'établir s'il existe une classe d'individus qui ne font pas l'expérience des émotions sociales mais qui sont autrement normaux, c'est-à-dire qui n'ont pas de retard mental ou qui ne sont pas autistiques. Il semble qu'une telle classe existe, qui contient ceux que l'on nomme les "sociopathes". Linda Mealey (1995) écrivait tout récemment à leur sujet :

Qu'ils soient criminels ou non, les sociopathes exhibent typiquement ce qui est considéré généralement comme un comportement irresponsable et peu fiable; leurs attributs incluent l'égocentrisme, une incapacité à former des engagements personnels à long terme et un degré marqué d'impulsivité. Sous un vernis superficiel de sociabilité et de charme, les sociopathes sont caractérisés par un déficit dans le domaine des émotions sociales (amour, honte, culpabilité, empathie et remords). D'un autre côté, ils ne sont pas intellectuellement handicapés, et sont souvent capables de tromper et de manipuler les autres à travers des arnaques et ruses élaborées, ou de commettre des crimes qui exploitent la confiance et la coopération des autres, comme la fraude, la bigamie et le détournement de fonds. Le sociopathe est "conscient de la contradiction entre son comportement et les 
attentes sociales, mais il ne semble ni guidé par la possibilité d'une telle contradiction, ni préoccupé par son occurrence". (p. 523 ; je souligne) $)^{9}$

Selon Frank, le problème des sociopathes vient de ce qu'ils ne développent pas les compétences émotionnelles comme l'empathie ou l'intérêt pour la détresse des autres et qu'à cause de cette anormalité dans le développement, ils n'acquièrent pas de sentiments moraux. Fait intéressant, si les jeunes sociopathes sont incapables d'empathie, ils semblent tout de même posséder une théorie de l'esprit (c'est-à-dire qu'ils peuvent attribuer aux autres des croyances et désirs différents des leurs) qui leur permet, avec un certain bonheur, de prédire et manipuler à leur avantage le comportement des autres. Les sociopathes sont également impulsifs, c'est-à-dire qu'ils sont incapables de transposer les coûts actuels d'une activité en gains futurs, ce qui explique pourquoi ils sont incapables de s'engager ou de coopérer, puisqu'ils agissent principalement sous l'impulsion d'émotions fondamentales comme la joie ou la colère. Cela laisse penser que le fonctionnement des centres responsables de ces émotions fondamentales est intact et que d'autres centres sont nécessaires à la production des émotions sociales. D'après Brothers (1995) et Barratt et Gardner (1995), le cortex orbifrontal postérieur joue un rôle important dans la production des émotions sociales. La destruction de ce centre, qui est lié entre autres à l'amygdale, résulte en difficultés de contrôle de l'impulsivité et en ce que Damasio et al. (1990) ont qualifié de "sociopathie acquise ${ }^{10}$. Damasio soutient en effet que la prise de décision des sujets humains est guidée par un ensemble de marqueurs somatiques positifs ou négatifs qui leur procurent une "sensation viscérale " concernant le choix d'une option donnée et attirent leur attention sur les conséquences négatives ou positives de leur action. Comme il l'écrit :

La prise de décision compétente dans le domaine social ne dépend pas seulement des conventions sociales et de l'éthique, et de la perception et de l'intelligence nécessaires pour manipuler de tels savoirs dans une situation de

9. Mealey propose également l'existence d'individus dont la sociopathie n'est pas la résultante d'une quelconque pathologie, mais qui présentent néanmoins les symptômes de la sociopathie. Selon elle, cette forme de sociopathie acquise résulte d'un choix d'une stratégie comportementale possible dans le contexte de coopération où les coopérateurs ne peuvent pas se permettre le luxe d'être des détecteurs parfaits des tricheurs (cette stratégie, comme le prédit la théorie des jeux évolutionnistes (Maynard Smith 1994), ne peut être adoptée que par une minorité d'acteurs sociaux, sinon l'édifice coopératif complet s'écroule). Si tel est le cas, leur existence viendrait étayer la thèse de Griffiths (1997) selon laquelle l'esprit humain, comme certains traits physiques (la couleur des yeux), est polymorphique et non monomorphique comme le suggèrent parfois les tenants de la PE. Rien, en effet, dans la théorie évolutionniste ne nous force à postuler que les mécanismes psychologiques humains sont monomorphiques.

Io. Le terme est cependant trompeur puisque ces sociopathes n'exhibent pas les tendances criminelles ou à la manipulation des véritables sociopathes. Les sociopathes du genre dont parlent Damasio et ses collègues constituent en fait des proies de choix pour les véritables sociopathes. 
la vie réelle. Il apparaît qu'un facteur somatique intervient tôt dans le processus, et se manifeste lui-même comme un état somatique "signal". [...] Dépourvus d'un marqueur somatique accordé aux conséquences futures d'une option donnée, les individus ont une probabilité accrue de faire leurs choix à partir des conséquences immédiates de ces options. Ils ont plus de probabilités de s'engager dans des comportements immédiatement gratifiants, mais qui, en fin de compte, seront sanctionnés par des punitions. (1993, p. 134)

Blair (1995) suggère une explication plus complète de la raison pour laquelle ces marqueurs somatiques ne se mettent parfois pas en place. Selon lui, la sociopathie est provoquée par le malfonctionnement de ce qu'il nomme le "Mécanisme d'Inhibition de la Violence " (MIV). Ce mécanisme est l'analogue de celui qui contrôle l'agression chez certains animaux sociaux. Par exemple, les chiens cessent l'attaque lorsque leurs conspécifiques exhibent les comportements typiques de la soumission (comme lorsqu'ils font la révérence (bowing)). C'est le même mécanisme qui est activé plusieurs fois pendant le jeu entre chiens et qui permet que celuici ne dégénère pas (Allen et Beckoff, 1998). Selon Blair, le MIV est « un mécanisme cognitif qui, lorsqu'activé par des signaux non-verbaux de détresse (par exemple, une expression faciale de tristesse, la vision et le son de pleurs), initie une réponse de retrait ; un schéma sera activé qui prédisposera l'individu à cesser l'attaque » $(1995, \text { p. 3 } 3)^{11}$.

Selon lui, le MIV est un prérequis pour le développement de trois aspects de la moralité : les émotions sociales, l'inhibition de l'action violente et la distinction entre les règles conventionnelles et morales ${ }^{12}$. Or, il appert que les sociopathes ne réagissent pas à la détresse des autres (par exemple, aux photographies de gens qui souffrent), ne développent pas d'empathie pour eux (au sens où ils ne développent pas "de réponses émotionnelles à la représentation d'un état interne de détresse d'un autre »(Blair, 1995, p. 4)) et qu'ils ne font pas la distinction entre règles morales et conventionnelles, traitant les premières comme des instances des dernières (quand on leur demande pourquoi il n'est pas bien de voler les autres, ils feront référence au fait que c'est parce que ça va à l'encontre de la règle, mais pas au fait que cela entraîne des conséquences sur le bien-être des autres). Le problème des sociopathes est donc que, le MIV étant inactivé, le développement normal ne peut suivre son cours et l'acquisition des émotions morales et de l'empathie est entravée. Cette situation laisse ces sujets uniquement avec les émotions fondamentales pour guider leur comportement, avec les conséquences que l'on connaît.

Terminons cette section par une remarque au sujet du type d'émotions que nous venons de mettre au jour. L'amour, la honte, la jalousie, si elles sont des émotions pour lesquelles ont peut donner une explication évolutionniste,

I I. Le MIV n'est pas le seul mécanisme contrôlant le comportement ; d'autres, comme le système de supervision attentionnelle, peuvent aller à l'encontre de ses outputs.

I2. Les règles conventionnelles sont celles que l'on peut violer sans problème («les garçons ne peuvent pas porter de robes") et les règles morales sont celles dont la violation entraîne une conséquence négative ou indésirable chez autrui ( " on ne doit pas voler ce qui appartient à autrui »). 
ne semblent pas pour autant pouvoir être analysées en terme de programme d'affects. Leurs effets ne sont pas nécessairement immédiats, leur déploiement peut s'étendre dans le temps et il résulte dans la mise en place de stratégies comportementales non-stéréotypiques à moyen terme, aussi vaudrait-il probablement mieux parler avec Griffiths (1997) de "patterns irruptifs de motivation " plutôt que de programmes. La jalousie, par exemple, rend un sujet plus attentif à certains stimuli dans son environnement qui pourraient être des indices de l'infidélité de sa compagne ou son compagnon; elle donne une nouvelle saillance à certains épisodes remémorés ; elle conduit le sujet à s'engager, selon son tempérament, dans des comportements variés allant de l'espionnage à la violence physique ; finalement son déroulement est accompagné par l'expression de différents types d'émotions allant de la colère à la peine. Ces émotions semblent également pénétrables cognitivement, comme le montrent les travaux de Frank et al. (1993), où des économistes se transforment, l'instant d'échanges sans lendemain, en sociopathes. Les comportements qu'elles commandent sont également relativement plastiques : certaines cultures prescrivent la violence physique et la séclusion comme remèdes à la jalousie, alors que d'autres cultures proscrivent ces mêmes actions (toutefois, à un niveau formel, il est possible d'établir une équivalence fonctionnelle entre les différents comportements : par exemple, ils ont tous le même effet). Elles sont finalement construites (selon toutes vraisemblance) à partir des émotions fondamentales et ne peuvent pour cette raison en être (la nature de la construction reste cependant à préciser : mélange (Ekman 1998), subsomption (Damasio 1993), extension (Rozin et Fallon 1987)). Ce serait donc une erreur de vouloir subsumer les deux types d'émotions sous un même terme ou domaine sous prétexte que l'on peut donner une explication fonctionnelle identique de chacune. Griffiths $(1994,1997)$ propose d'étudier les traits phénotypiques (physiques ou psychologiques) en utilisant plutôt les outils de la cladistique, c'est-à-dire en utilisant la représentation arborescente des relations de descendance ou d'ascendance entre organismes. Selon lui, le fait que les émotions fondamentales soient partagées par un vaste groupe d'espèces, comme nous l'avons montré, permet d'effectuer un certain nombre de généralisations à leur propos, de les traiter comme une espèce naturelle. Les émotions sociales, d'un autre côté, sont apparues récemment et ne sont probablement que l'apanage de quelques primates (De Waal 1997) et des humains. Pour cette raison, on ne peut assumer qu'elles possèdent les mêmes propriétés que les émotions fondamentales (par exemple, l'automaticité ou le fait qu'elles sont desservies par des structures cérébrales primitives comme l'amygdale) ou qu'elles peuvent être expliquées en invoquant la même structure causale sous-jacente. 


\section{Constructionnisme social}

Notre thèse a jusqu'ici consisté à soutenir que les émotions sont sociales en deux sens : dans un premier sens, les expressions émotionnelles peuvent servir à la coordination intraspécifique; dans un deuxième sens, certaines émotions peuvent être dites sociales parce qu'elles permettent l'engagement dans la coopération à long terme qui est le nerf de la socialité humaine. Je crois que tout comme les émotions que sont la peur ou la joie, les émotions sociales sont jusqu'à un certain point panculturelles (je propose certains qualificatifs à cette position dans la section qui suit) ${ }^{13}$. Cette opinion se heurte toutefois à une forte opposition de la part des constructionnistes sociaux. Les tenants du constructionnisme social ${ }^{14}$ soutiennent en effet que les émotions sont "socioculturellement constituées ", que le langage sur les émotions n'exprime pas une réalité préexistante, mais la crée. Rom Harré, qui est un des principaux avocats du constructionnisme dans sa version forte, s'oppose au réalisme qui sous-tend l'approche naturaliste. Selon lui,

Les psychologues se sont toujours débattus contre l'illusion persistante que dans des études comme celles des émotions il y avait quelque chose là [something there], l'émotion, duquel le mot "émotion" n'est qu'une simple représentation. (1986, p. 4)

Les émotions sont donc des produits sociaux dont les constituants principaux sont des croyances et des jugements dérivés de la culture. Elles sont constituées et analysables en termes de ce que Armon-Jones (1986a) a nommé des croyances et jugements non-naturels, c'est-à-dire des états mentaux dont le contenu est déterminé par les systèmes culturels de croyances ainsi que par les normes et valeurs des communautés particulières ${ }^{15}$. De plus, selon les constructionnistes, les émotions ne sont pas des réponses naturelles, mais des ensembles d'expériences et d'expressions déterminés socialement (des "rôles transitoires ", comme les nomme Averill), qui sont acquis, qui reçoivent une interprétation en terme de passion plutôt que d'action et qui figurent de façon stratégique dans les situations sociales. En d'autres mots, les émotions sont des rôles transitoires qui sont prescrits socialement aux individus dans certaines situations, un peu comme les différents rôles de père, enseignant, frère ou partenaire sportif que nous empruntons dans une journée (rôles pour lesquels, d'ailleurs, certaines émotions sont prescrites). Comme les rôles qu'empruntent les acteurs, les émotions sociales sont des "faire comme-si » ("pretence ») socialement

I3. Keltner et Buswell (1996) soutiennent à l'appui de cette idée que les antécédents et les expressions de l'embarras et de la honte sont panculturels.

I4. Dont les principaux défenseurs sont Armon-Jones, 1986a et b ; Averill, 1980, 1986 ; Greenwood, 1992a et b ; Harré, 1986 ; Lutz, 1982, 1986 ; Lutz et White, 1986.

I5. Certains constructionnistes soutiennent que la fonction des émotions est la régulation des comportements indésirables et la promotion des attitudes qui reflètent et endossent les pratiques religieuses, politiques et sociales d'une communauté (Armon-Jones, 1986b). Les émotions seraient ainsi une forme d'inscription dans la chair des micro-pouvoirs. Je ne discuterai pas cette thèse dans ce qui suit. 
construits ou induits. Sous cet angle, elles sont similaires aux cas de possession démoniaque, d'hystérie ou d'enlèvement par des extra-terrestres : leur réalité sociale précède leur réalité psychologique, elles sont des modèles auxquels se conforme le sujet ${ }^{16}$.

Pour illustrer cette thèse, prenons l'exemple de la honte. La honte n'est pas, selon les constructionnistes, une expérience que nous découvrons en nous, comme on peut découvrir la douleur par exemple (Lutz (1986) montre d'ailleurs que cette conception de l'émotion comme à l'intérieur n'est pas commune à toutes les cultures ; voir Dumouchel 1996 pour une tentative de remplacer cette conception privée de l'émotion par une conception publique). Nous devons apprendre à être honteux, c'est-à-dire que nous devons apprendre à accepter une évaluation, dérivée socialement, d'une certaine classe d'actions et de situations comme personnellement dégradante et humiliante. Ce qu'est une situation dégradante et humiliante est quelque chose qui est déterminé conventionnellement, ce n'est pas une propriété physique de la situation (ce qui peut être humiliant pour moi peut être une source de fierté pour un autre). Nous devons également apprendre à réagir vis-à-vis cette situation d'une façon appropriée, par exemple, en exprimant un repentir sincère. En d'autres mots, chaque culture dicte aux sujets une façon de construire les situations et ce qu'ils devraient ressentir et faire dans ces situations ${ }^{17}$. Cette théorie, en mettant l'accent sur les croyances, les désirs et les jugements culturels a des conséquences sur les classes de systèmes auxquelles il est possible d'attribuer des émotions. Par exemple, l'attribution d'émotions aux animaux ne peut, dans ce contexte, qu'être métaphorique puisqu'ils n'ont pas de langage ni d'institutions culturelles et sociales semblables aux nôtres. La même conclusion s'applique aux enfants. On ne peut guère leur attribuer des émotions comme la jalousie ou la honte avant qu'ils n'aient intériorisé les normes et attitudes culturelles qui constituent

16. Le fait que l'on soit en face d'un «faire comme-si " n'implique pas que le sujet de l'émotion sait qu'il fait semblant, tout comme ceux qui souffrent du Syndrome de Personnalité Multiple (SPM) ne savent probablement pas qu'ils ne font que se conformer à une image socialement constituée. Cela n'implique pas non plus qu'il n'y ait aucun effet physiologique concomitant (les sujets peuvent confabuler sur l'origine de cet effet ou bien il peut être induit par la prescription sociale), mais pour les constructionnistes cet effet n'est pas nécessaire.

I7. De Sousa (1980) résume bien ce qui est en jeu pendant l'apprentissage pour un constructionniste : les enfants apprendraient à "gestalter " les situations en termes de "scénarios paradigmatiques" (p. 142), scénarios qui seront plus tard raffinés par l'expérience et étendus à de nouvelles situations. De Sousa reprend ici le modèle kuhnien de l'éducation du scientifique qui consiste à produire une façon de voir le monde ainsi que l'idée que le développement de la science normale consiste simplement en un raffinement de cette façon de voir le monde. On peut donc penser que l'apprentissage des catégories évaluatives ainsi que celle des comportements appropriés est l'affaire d'un entraînement qui ne se termine pas nécessairement avec la fin de l'enfance, mais pourrait plutôt s'étendre sur toute la vie. Le développement adulte consisterait plus particulièrement à affiner l'évaluation des situations, à devenir plus expérimenté dans la façon d'exprimer les émotions et plus sophistiqué dans l'attribution d'émotions à soi-même ainsi qu'aux autres. 
l'émotion. Les syndromes émotionnels présupposent le développement préalable des capacités cognitives non émotionnelles (comprendre que quelqu'un agit intentionnellement, par exemple).

Les émotions reposent donc sur un ensemble de croyances et de jugements, ou ce que d'Andrade (1987, p. 113) nomme un "modèle culturel ». Elles sont donc holistiques en ce sens, ou, pour adopter la distinction de Searle (1995), elles sont ontologiquement subjectives et épistémologiquement objectives (elles existent bel et bien dans le monde, mais requièrent un arrière-plan de pratiques humaines pour exister). Ce modèle (ou théorie) est constitué de règles qui dictent la façon dont les émotions doivent être expérimentées et exprimées, mais qui définissent également les catégories de stimuli qui peuvent déclencher différentes émotions ${ }^{18}$.

Les émotions dépendent d'abord et avant tout de l'évaluation de la situation par l'individu. Elles dépendent donc des croyances de celui-ci à propos de la situation. Elles sont donc intentionnelles, ce qui les distingue des sensations de douleur par exemple. Pour les distinguer des croyances ordinaires (cold cognitions), les constructionnistes doivent mentionner que ces croyances sont hautement évaluatives, au sens où elles comportent un jugement sur la situation (désirable ou indésirable), par opposition à des jugements sur ce qui est vrai ou faux. Elles sont également personnelles, au sens où ces croyances nous concernent ou mettent en cause notre identité personnelle. Par exemple, si je crois que quelqu'un devrait être honteux, je ne deviens pas, moi-même, honteux (à moins que ce ne soit un proche et que je me sente en quelque sorte responsable pour lui).

Si les croyances jouent un rôle central dans la constitution de l'émotion, il devient possible de postuler l'existence d'une réelle diversité ontologique dans le domaine des émotions. Puisque les émotions sont socialement constituées par des évaluations qui reflètent l'ordre moral propre à chaque culture, on peut faire l'hypothèse que les cultures qui diffèrent par leur ordre moral, différeront également par le répertoire d'émotions que les individus pourront expérimenter. Prenons, pour illustrer cette thèse, l'exemple de l'accidie (Harré et Finlay-Jones 1986), émotion disparue aujourd'hui, mais courante dans les monastères au sixième siècle et qui fut, un temps, le huitième péché capital ${ }^{19}$. Elle est constituée par une forme de représentation des actions propres aux moines. Cette représentation implique une forme de paresse dans l'accomplissement des

I8. Averill (1986) distingue quatre types de règles, les règles d'évaluation (qui déterminent le contenu intentionnel des émotions), les règles comportementales (qui déterminent la façon dont les émotions doivent être exprimées), les règles de prognosis (qui déterminent le cours temporel et la progression des émotions) et les règles d'attribution (qui déterminent la façon dont une émotion doit être expliquée).

I9. Cette dépendance vis-à-vis d'un ordre moral particulier a pour conséquence qu'avant de pouvoir comprendre une émotion, il est nécessaire de comprendre le système socio-culturel dans lequel elle s'inscrit. Comme le note White (1993), le fait de se concentrer sur ces systèmes fait que l'on cesse de regarder les émotions comme privées, intérieures et individuelles pour les voir plutôt comme publiques, extérieures et sociales. Voir Heelas (1986) et Lutz (1986) pour des exemples d'émotions dans des cultures non occidentales. 
devoirs spirituels ainsi que des sentiments de misère ou de chagrin (et non pas de culpabilité et de honte). L'accidie est un "appauvrissement de l'âme ", genre de "burnout ", qui résulte de l'abrutissement du moine qui cesse d'être conscient de lui-même comme d'une créature divine ou possédant une dimension spirituelle (Bartlett 1990). L'accidie ne pouvait pas être surmontée par l'exécution sans entrain de ses devoirs, elle l'était seulement lorsque celle-ci était accompagnée de plaisir. Cette émotion ne pouvait exister que dans un ordre moral particulier où un sujet avait des devoirs spirituels vis-à-vis Dieu. Nous sommes toujours en proie à la procrastination et à la paresse, note Harré, mais les émotions qu'elles provoquent ne sont plus les mêmes, puisqu'elles sont maintenant définies sur un arrière-plan moral différent, soit l'éthique de la production ${ }^{20}$.

Cette hypothèse de la diversité ontologique des émotions suppose une grande malléabilité au niveau neuronal. Averill (1982) affirme, par exemple, que la théorie neuropsychologique qui découle de l'analyse constructionniste est une théorie qui met l'accent sur la plasticité des circuits neuronaux, par opposition aux circuits pré-câblés de Panskeep. Il soutient ailleurs (1986, p. 101) que les milliers d'années d'évolution comme hominidé ont sûrement rendu plus facile à l'homme l'apprentissage de certaines choses, mais que les systèmes béhavioraux qui ont survécu à cette évolution sont organisés de façon lâche et qu'ils peuvent être transformés et combinés d'une infinité de manières.

Notons enfin que pour les constructionnistes, les aspects physiologiques des émotions jouent un rôle secondaire (pour certains, ils ne sont même pas nécessaires), n'étant en quelque sorte que des effets secondaires de phénomènes socio-culturels plus fondamentaux (cf. Harré, 1986, p. 5, ainsi que Armon-Jones, 1986a, p. 36). Le programme constructionniste est plus ou moins schactérien au sens où il admet à la suite de Schacter (Schacter et Singer 1962) le rôle déterminant de la cognition et des jugements dans la constitution des expériences émotionnelles ${ }^{21}$.

\section{Le concept populaire d'émotion à la lumière du naturalisme et du constructionnisme}

J'avais noté au début de ce texte que certains perçoivent une tension entre l'approche naturaliste et l'approche constructionniste et que cette tension proviendrait non seulement de l'utilisation de méthodes différentes ou d'une focalisation de l'attention sur différents aspects des émotions, mais également

20. Dans ce qui me semble être une réfutation de la thèse constructionniste, Finlay-Jones maintient que l'accidie existe encore aujourd'hui chez les chômeurs ou les femmes au foyer (voir également Bartlett (1990) qui soutient qu'elle existe plutôt chez les travailleurs qui négligent de s'investir ailleurs que dans leur travail).

2I. Il s'éloigne parfois de la position de ce dernier en ne considérant pas l'activation physiologique comme une condition nécessaire à la présence d'une émotion. 
de ce qu'on croit généralement que les résultats de chacune sont incompatibles entre eux. J'aimerais démontrer que cette perception est erronée.

Tout d'abord, plusieurs croient que la diversité des émotions mise au jour par les constructionnistes est irréconciliable avec l'existence des programmes d'affects. C'est mal comprendre l'approche naturaliste que de la confondre avec une position qui maintiendrait que les émotions (comme processus) sont des programmes fermés, imperméables à l'apprentissage. Au contraire, comme je l'ai montré plus haut, les mécanismes d'évaluation qui déclenchent les programmes d'affects sont relativement souples. La prise en compte de la neurologie des émotions nous permet de penser que des concepts abstraits ou des situations ou contextes appris socialement peuvent également en venir à déclencher les programmes d'affects. On l'a dit, le conditionnement ouvre à de nouveaux environnements le canal qui liait certains stimuli aux réponses programmées. Les naturalistes montrent également que les réponses peuvent être tempérées ou complètement masquées par les règles d'expression, qui sont apprises culturellement. La position évolutionniste suppose donc une certaine malléabilité du système émotionnel, mais elle n'est pas prête à lui reconnaître une plasticité totale. Elle postule l'existence d'un répertoire de base sur lequel des variations peuvent être exécutées. Comme l'écrit Ekman,

Mon attention [porte] sur les arguments et l'évidence qui suggèrent que les émotions sont le produit de notre évolution [...] Cette position ne nie pas le rôle important de la culture et des processus d'apprentissage social dans chaque aspect de l'émotion - le contrôle des expressions, la représentation symbolique de l'expression émotionnelle, la façon dont quelqu'un évalue les situations pertinentes aux émotions, les attitudes à propos de ses propres émotions, ainsi de suite - mais ce n'est pas un système totalement malléable. Il y a des contraintes résultant de notre évolution [...] (1992a, p. 550)

La situation est donc la suivante : on peut s'attendre à ce que les humains exhibent les mêmes tendances émotionnelles, qu'ils disposent des mêmes mécanismes associatifs préparés; d'un autre côté, comme les constructionnistes l'ont bien montré, chaque culture conceptualise le champ affectif à sa façon. Il faut donc que le système émotionnel dispose d'une certaine plasticité. Je crois qu'une des meilleures façons de concilier les deux approches est ce que l'on a nommé la théorie du clavier (keyboard theory), c'est-à-dire que l'on suppose que chaque société disposerait d'un même clavier émotionnel, mais différerait quant à sa manière d'en jouer (Levy 1984). Contrairement à ce qu'affirmait Harré, il y aurait donc un "something there » biologique qui existe au même titre que l'ensemble des pratiques sociales auxquelles il fait référence. Dans ce cas, il est possible de comprendre que ce que démontrent les constructionnistes sont les trois choses suivantes :

1) Premièrement, on constate que les attitudes concernant les mêmes émotions et leur théorisation diffèrent entre les cultures. Par exemple, les Inuit Utku considèrent la colère comme une émotion infantile et en découragent l'expression, alors que les Américains y voient plutôt une marque de caractère et l'encouragent. Contrairement à ce qu'affirmait 
Solomon, ceci n'équivaut pas à dire que l'émotion de colère n'existe pas chez les Inuit, mais seulement que son expression est inhibée comme résultat d'un processus d'acculturation. Solomon le reconnaît d'ailleurs, implicitement du moins, puisqu'après avoir dit que les Inuit ne se mettent jamais en colère, il écrit que chez ces derniers la colère est rare et crainte (1984 p. 243). Donc elle existe, elle peut être rare, soit, mais elle existe quand même ${ }^{22}$. Pour une raison quelconque, une culture peut décider de crier haro sur une émotion, mais cette pratique ressemble à celle des Japonais qui plaçaient les pieds des fillettes dans des bandages pour qu'ils restent menus. Le potentiel émotionnel est là, son utilisation est simplement découragée.

2) Deuxièmement, les constructionnistes montrent non seulement que les différentes cultures ont des attitudes différentes concernant les expressions d'émotions ou les objets dignes de l'expression, mais qu'elles entretiennent également des théories différentes sur les émotions, par exemple sur ce qui les cause, sur leur source ou leur dynamique. Certaines cultures placent le lieu des émotions dans le foie; d'autres, comme les anciens Grecs, le plaçaient dans le comportement (Heelas, 1986). Ces théories ne modifient pas le phénomène. Averill, dans un texte sur l'amour romantique, résume cette idée en utilisant la métaphore suivante :

Une société peut concevoir le tonnerre et les éclairs comme des manifestations de la colère de quelque dieu; mais une telle conception, qu'importe sa signification idéologique, n'altérerait pas de façon fondamentale les principes météorologiques qui rendent compte des orages. N'est-il pas possible également que l'amour soit un principe de la nature humaine, et que tout ce qui varie soit la façon dont l'amour est conceptualisé et utilisé ? (1985, p. 94 ; voir également Stearns, 1993)

Dans le même ordre d'idées, chaque culture diffère dans l'importance qu'elle accorde à chaque émotion. Les Tahitiens, par exemple, ne possèdent pas de mot pour désigner la tristesse, l'esseulement et la dépression. Ils ressentent bien une sensation de fatigue ou de lourdeur, mais ils interprètent cette sensation de façon non émotionnelle. Ils la conçoivent comme une maladie ou comme l'effet d'un esprit. Levy (1984) a proposé une distinction pour rendre compte des différences entre cultures : on parlera d'« hypocognizance » ou d' "bypercognizance » au sujet d'un domaine particulier d'émotions selon l'état plus ou moins développé de théorisation de ce domaine dans une culture. Comme le montre l'exemple de Levy, les Tahitiens ne disposent peut-être pas de mots pour décrire leur état, ils n'en

22. De toute façon, les évidences sur lesquelles s'appuyait Solomon pour faire cette affirmation semblent être réfutées par des observations récentes. Par exemple, Stearns et Stearns écrivent : "Briggs a décrit la façon dont la tribu des Inuit Utku désapprouvait la colère et cherchait à la supprimer à travers un processus de socialisation. Mais l'étude n'a pas prouvé que les Utku sont des gens sans colère. Ils boudent régulièrement lorsqu'ils sont mis au défi (colère tournée vers soi ?) et ils battent leurs chiens et en abusent d'autres façons. » (cité par Oatley, 1993, p. 342) 
font peut-être pas une émotion, mais ils font tout de même l'expérience de la tendance émotionnelle. Ne pas disposer de mot pour décrire un phénomène n'implique en rien son inexistence (pace Whorf).

3) Troisièmement, les constructionnistes montrent également que les stimuli qui déclenchent les émotions demandent parfois des habiletés cognitives élevées : par exemple, la compréhension de la hiérarchie sociale ainsi que des règles morales particulières d'une communauté. En raison de cette exigence, l'apprentissage de certaines émotions ne peut se faire que sur une longue période et l'attribution d'une émotion à un enfant ne peut se faire qu'après qu'il ait assimilé une grande quantité de connaissances sur l'organisation sociale dans laquelle il évolue. Par exemple, l'apprentissage de la peur des dieux (ou de toute autre peur non naturelle ; cf. Armon-Jones, 1986b, pp. 62-67) sera un processus de socialisation qui s'étendra sur une bonne partie de l'enfance. Une émotion comme la peur des dieux demande de la part de l'enfant la connaissance de certaines normes, les conséquences de la transgression de ces normes et la croyance en l'existence des dieux.

J'aimerais dire deux choses au sujet de ce type de peurs.

(a) Le fait qu'une émotion ou que la variation d'une émotion (le dégoût moral, par exemple) ne fasse pas partie du répertoire de l'enfant à la naissance ne pose pas problème à un évolutionniste; après tout, dire d'une émotion qu'elle possède une explication évolutionniste n'implique rien quant à l'histoire de son développement, au moment où elle doit apparaître dans le développement ou même à la façon dont elle doit être construite. En effet, dans le domaine de la physiologie, il y a des caractères qui ne sont pas présents à la naissance, mais qui apparaissent plus tard, comme les seins ou la barbe. Les travaux de Chomsky indiquent que la même chose est vraie dans le domaine du langage. Il y a des capacités qui demandent le développement d'autres capacités avant de pouvoir être exercées. La même morale semble s'appliquer aux émotions. Kagan explique par exemple le fait que la détresse de la séparation apparaisse chez tous les enfants vers le septième mois de leur vie par le fait qu'elle nécessite la maturation de la capacité à recouvrer de la mémoire le souvenir des visages et à les comparer avec les visages actuels, capacité qui n'est pas présente à la naissance. Dans le même ordre d'idées, il est tout à fait possible que certaines émotions ne puissent apparaître avant que l'enfant ait développé sa capacité d'attribuer des intentions différentes des siennes aux autres. Une fois cette capacité développée, il peut attribuer aux autres des intentions qui ne sont pas les siennes, comme l'intention de lui causer du tort. Il peut également se sentir honteux d'avoir tenté de tromper les autres ${ }^{23}$. Il peut donc comprendre les

23. Selon les observations de Frith (1989), les autistes qui, selon l'hypothèse en vogue, ne possèdent pas cette capacité ne développent pas de sentiment de honte ou de culpabilité. Il semble cependant que l'histoire soit plus complexe puisque Blair (1996) a montré que les autistes faisaient la distinction entre transgression morale et conventionnelle qui est un des marqueurs du développement du MIV. Ils auraient donc probablement ces émotions. Ceci serait consistant avec l'hypothèse de De Waal (1997), qui soutient que les chimpanzés ont ce type d'émotions en dépit du fait qu'ils n'ont pas une théorie de l'esprit à proprement parler. 
intentions derrière les actions ou du moins en imputer à autrui. Enfin, les données provenant de la psychologie du développement (Lewis 1993) montrent assez clairement que toutes les émotions fondamentales sont en place vers le septième ou huitième mois, que d'autres émotions comme l'embarras et l'envie apparaissent avec le développement de la conscience de soi vers deux ans et demi, alors que les émotions comme l'orgueil et la honte n'apparaissent qu'avec la capacité à acquérir des règles et des standards (les tabous sociaux, comme le fait que jouer dans son nez est malpropre et que l'on ne doit pas le faire devant les autres) vers la troisième année.

(b) Le fait que certaines évaluations sociales fassent partie de l'émotion n'implique pas que l'émotion est complètement constituée socialement. Comme le notent Fisher et Fridja (1992 ; voir également Gibbard 1993, p. 67), ces évaluations pourraient résulter de sensitivités innées. Par exemple, disent-ils, il pourrait très bien exister une sensibilité émotionnelle innée au cas où quelqu'un dévie des normes d'un groupe. Se faire indiquer que l'on dévie des normes peut créer une sorte de détresse non apprise, même si les normes sont des produits sociaux.

Une des sources de la tension entre les naturalistes et les constructionnistes provient de leur conception respective (et également fautive) du développement. Les naturalistes supposent souvent que les émotions, parce qu'elles sont universelles, sont également innées (ou bien produites par un programme développemental disjonctif, c'est-à-dire un programme qui peut entrer seulement dans un certain nombre d'états prédéterminés dépendants des variations dans les conditions environnementales). Les constructionnistes, s'intéressant plutôt à la diversité, supposent plutôt que les émotions sont apprises et construites socialement. Griffiths et Gray (1994) proposent un modèle du développement qui permet de résoudre les problèmes générés par la dichotomie inné/acquis : celui de la " construction hétérogène ". "L'expression "construction hétérogène" ", comme ils l'écrivent, "est conçue pour convier l'idée que le phénotype psychologique est construit à travers l'interaction de facteurs "biologiques" traditionnels, de facteurs "culturels" traditionnels et de facteurs qui sont difficiles à classifier dans les termes de cette dichotomie " (Griffiths et Gray, 1994, p. 132). L'imprinting est un exemple de construction hétérogène : certains oiseaux n'apprennent apparemment à chanter que s'ils sont exposés à un type de chant particulier pendant une période de leur développement (Gould et Marler 1987). La stabilité transgénérationnelle du chant chez ces oiseaux doit être expliquée en mentionnant leur "instinct d'apprentissage ", mais également le fait que les conditions environnementales dans lesquelles les oisillons grandissent sont relativement constantes; par exemple, ils grandissent entourés d'oiseaux de la même espèce (selon Griffiths et Grey, c'est l'ensemble des conditions biologiques et environnementales, qu'ils nomment "systèmes développementaux ", qui est l'objet de la sélection). On peut penser que la communauté humaine offre des conditions environnementales 
relativement stables qui permettent la mise en place d'émotions universelles, mais également des conditions changeantes qui influencent l'expression des phénotypes psychologiques. Les programmes d'affects seraient le résultat d'une construction où les facteurs «biologiques " joueraient un rôle plus important que les facteurs "culturels", alors que les émotions sociales seraient produites par une interaction plus équilibrée des facteurs " biologiques » et « culturels » (dans ce cas de l'organisation sociale humaine, mais également de la culture proprement dite).

Il est également possible de voir le débat entre les naturalistes et les constructionnistes comme portant sur la nature de l'explication adéquate du phénomène émotionnel. Pour bien comprendre ce débat, j'introduirai une distinction. Armon-Jones (1986a) propose de distinguer deux thèses constructionnistes : la thèse forte et la thèse faible. Selon la thèse forte, les émotions étant constituées de croyances et de jugements produits par l'environnement socio-culturel, il n'existe d'émotion que sociale. L'idée même d'émotion naturelle est, par définition, impensable. Il existe cependant une thèse faible, plus plausible, qui concède aux naturalistes l'existence d'émotions naturelles, non touchées par l'influence socio-culturelle. Cette thèse faible a l'avantage de pouvoir expliquer pourquoi, dans certains cas de peur, les humains exhibent des comportements semblables à ceux des animaux. Pour celui qui adhère à la thèse faible, il reste à expliquer le lien entre les émotions naturelles et les émotions socio-culturelles. La plupart des naturalistes soutiennent que les émotions socio-culturelles sont des variations à partir des émotions biologiques de base. Mais, selon ArmonJones, le lien n'est pas aussi simple :

L'argument crucial ici est que la prédication du terme "émotion" à la fois aux émotions primitives et socialisées est justifiée par l'idée que les deux phénomènes sont suffisamment similaires pour être capturés par le même terme. L'objection du constructionniste faible à ce point de vue serait que, en autant que les émotions impliquent des attitudes spécifiquement socioculturelles, apparaissent dans des contextes spécifiquement socioculturels et entretiennent une relation prescriptive ou fonctionnelle à de tels contextes, le naturaliste ne peut extrapoler son explication des émotions naturelles pour les expliquer. (Armon-Jones, 1986a, p. 39 ; je souligne)

En effet, si le naturaliste peut expliquer adéquatement le noyau émotionnel, il n'a pratiquement rien à dire sur les variations de chaque société à partir de celui-ci. Armon-Jones croit en fait que le naturaliste ne peut expliquer pourquoi la peur est prescrite dans certains contextes non naturels et pas dans d'autres. Afin d'expliquer pourquoi dans telle société une expression émotionnelle est prescrite ou doit être dissimulée, on doit faire référence aux normes ou à l'organisation politique de cette société. Les émotions acquièrent dans ce contexte une nouvelle fonction qui n'est plus leur fonction première : elles deviennent gardiennes des valeurs d'une société. Par exemple, ce qui provoque la peur chez les Chewong est la transgression des règles de partage et de réciprocité dont cette société dépend pour sa survie. 
La converse est également vraie. Si certains naturalistes reconnaissent les limites de leur position (Panskeep, 1994), ils font remarquer en même temps que l'approche constructionniste laisse de côté une partie du phénomène. Panskeep, qui compare les systèmes de commande des émotions à des arbres avec des branches qui touchent les aires corticales, un tronc qui reflète les anciens noyaux exécutifs des systèmes et des racines qui plongent dans le tronc cérébral, écrit que

Même si les perspectives constructionnistes sociales peuvent faire des contributions substantielles aux plus hautes branches de l' "arbre" émotionnel, elles ne peuvent pas, de façon réaliste, s'occuper des questions fondamentales du tronc [basic "tronc-line" issues]. [...] l'expérience affective humaine émerge ultimement de systèmes neurosymboliques anciens du cerveau mammélien qui promeuvent inconditionnellement la survie, et si la psychologie veut se développer comme une science rigoureuse, elle doit fortement préconiser une analyse neurobiologique détaillée de ces systèmes d'opération du cerveau qui sont communs à tous les mammifères [...] Le constructionnisme social n'a pas besoin de polémiquer au sujet des questions de primauté. Il fonctionne mieux dans un domaine différent d'investigation [...] Les approches constructionnistes peuvent fournir des schémas de la façon dont les systèmes génétiquement équipés [genetically endowed] se développent complètement en interagissant avec la vaste complexité du monde réel. (Panskeep, 1992, p. 559)

Je crois que l'analyse de Panskeep est correcte pour certains cas : les naturalistes et les constructionnistes travaillent effectivement à des niveaux différents, les premiers se concentrant sur les universaux, les autres sur les différences entre cultures. Chacune des deux approches s'intéresse donc à des aspects différents des émotions, à l'apport de facteurs différents qui concourent tous à expliquer pourquoi les réponses émotionnelles ont la forme qu'elles ont. En un sens, il serait souhaitable de voir les deux approches comme complémentaires et participant à une explication multiniveaux de l'émotion (voir Oatley 1993).

\section{L'avenir du concept d'émotion}

Si la version faible du constructionnisme est compatible avec le naturalisme, sa version forte ne l'est pas. On sait que cette version soutient que l'activité physiologique n'accompagne que les versions extrêmes des émotions et qu'elle n'est pas une condition nécessaire à la présence d'une émotion. Lorsque l'activation est présente, elle est induite par la culture (la direction de la causalité est descendante) $)^{24}$. En ce sens, l'émotion telle que conçue par les constructionnistes forts ressemble plus à un phénomène comme l'effet

24. Un autre fait militant contre l'assimilation des objets des constructionnistes forts et des naturalistes est que les émotions qui sont décrites par ces derniers, même si elles supportent une certaine variance, $n$ 'en gardent pas moins une base commune (principalement due aux facteurs biologiques et environnementaux stables). 
placebo (où un effet thérapeutique est induit par un aspect autre que la cause identifiée par la théorie thérapeutique) ou comme la confabulation dans les cas de souvenirs présumément refoulés (où des sujets sont induits à leur insu à croire qu'ils ont été enlevés par des extra-terrestres ou qu'ils ont subi des sévices sexuels; Loftus et Ketcham 1994). Si on accepte l'idée que les constructionnistes forts réfèrent bien eux aussi à un sous-ensemble des phénomènes mentaux que l'on regroupe couramment sous le terme "émotion », il semble que l'on doive accepter le verdict de Rorty (1980, p.1): «Les émotions ne forment pas une classe naturelle ». En effet, à une extrémité, nous avons ces phénomènes dont les caractéristiques principales sont expliquées par la présence de programmes d'affects déclenchés par des systèmes modulaires; à l'autre, nous avons des états mentaux qui ne produisent qu'incidemment des effets physiologiques, qui sont des rôles que les sujets remplissent et dont la forme est expliquée complètement (ou en majeure partie) par des facteurs sociaux ; au milieu, nous avons ces émotions construites à partir, et en utilisant, des émotions fondamentales et qui sont relativement universelles soit à cause de facteurs biologiques ou à cause de la constance de l'environnement humain. Ces phénomènes n'ont pas grand chose en commun : ils n'ont pas la même fonction ; ils ne sont pas desservis par les mêmes structures neuronales; ils n'ont pas le même degré de pénétrabilité cognitive ; et finalement, leur explication cladistique est différente. Cette trifurcation est une bonne raison, selon Griffiths (1997), pour endosser une forme de scepticisme concernant l'utilité théorique du concept. Si cette représentation est adéquate, notre concept d'émotion devra peut-être être éliminé (ou révisé de façon substantielle) pour faire place à de nouveaux concepts plus aptes à rendre compte des caractéristiques des phénomènes mentaux que nous regroupons actuellement sous le même nom. Comme l'a montré Stich (1996) cependant, il n'existe pas d'argument allant de la fragmentation d'un concept ou d'une modification majeure de son extension et/ou de son intension à son élimination (pour un aperçu des arguments de Stich, voir Faucher et Deragon, 1999). Le concept d' « étoile » est un bon exemple de concept qui a survécu en dépit de révisions majeures de son intension et extension. La fragmentation d'un concept populaire n'est pas non plus sans précédent en psychologie : c'est ce qui est arrivé par exemple au concept de mémoire. Pendant des années, la mémoire a été conçue comme un phénomène cognitif unitaire auquel correspondait une seule structure neuronale. Cette conception a cependant été rejetée au cours des dernières années pour une autre beaucoup plus adéquate. Aujourd'hui la recherche postule l'existence de plusieurs genres de mémoire, chacune desservie par sa propre structure neuronale (mémoire à court terme, mémoire à long terme, procédurale, discursive, sémantique, épisodique, etc. ; voir Tulving (1995)). Il est assez difficile de prédire ce qui arrivera au concept d'émotion. Le fait que le concept d'émotion des constructionnistes forts semble décrire des phénomènes qui ont plus à voir avec l'induction de croyances ou de comportements qui semblent à leurs possesseurs naturels, serait sûrement une raison suffisante pour certains éliminativistes, comme 
Patricia Churchland (1988), de réclamer l'élimination du concept populaire d'émotion. Il n'appartient cependant pas au philosophe de déclarer unilatéralement la mort des concepts. Nous espérons cependant avoir démontré qu'une analyse attentive des phénomènes appartenant à l'extension du concept populaire d'émotion milite minimalement en faveur d'un réaménagement conceptuel majeur ".

\section{Bibliographie}

Allen, C. et Beckoff, M. 1997. Species of Mind: the Philosophie and Biology of Cognitive Ethology, Cambridge : MIT Press.

Armon-Jones, C. 1986a. "The Thesis of Constructionism ». In The Social Construction of Emotions, sous la direction de R. Harré, Oxford : Basil Blackwell, p. 32-56.

1986b. "The Social Function of Emotion». In The Social Construction of Emotions, sous la direction de R. Harré, Oxford : Basil Blackwell , p. 57-82.

Averill, J. R. 1980. "A Constructionist View of Emotions ». In Emotion: Theory, Research and Experience, sous la direction de R. Plutchik et $\mathrm{H}$. Kellerman, Vol. 1, p. 305-339.

1982. "Emotions : Hard- or Soft-Wired ?». Behavioral and Brain Sciences, vol. 3, p. 424.

1985. "The Social Construction of Emotion : With Special Reference to Love ". In The Social Construction of a Person, sous la direction de K.J. Gregen et K.E. Davis, New York : Springer-Verlag, p. 89-109. 1986. "The Acquisition of Emotions During Adulthood». In The Social Construction of Emotions, sous la direction de R. Harré, Oxford : Basil Blackwell, p. 98-118.

Barratt, E. et R. Gardner. 1995. "Sociopathy, Evolution, and the Brain ". Behavioral and Brain Sciences, vol. 18, no. 3, p. 544.

Bartlett, S. 1990. "Acedia: The Ethiology of Work-Engendered Depression ". New Ideas in Psychology, vol. 8, no. 3, p. 389-396.

Blair, R. 1995. "A Cognitive Developmental Approach to Morality: Investigating the Psychopath ». Cognition, 57, p. 1-29. 1996. "Brief Report : Morality in Autistic Child ». Journal of Autism and Developmental Disorders, vol. 26, no. 5, p. 571-579.

Brothers, L. 1995. "Neurophysiology of the Perception of Intentions by Primates ». In The Cognitive Neurosciences, sous la direction de M. Gazzaniga, Cambridge : MIT Press, p. 1107-1115.

* J'aimerais remercier Paul Dumouchel, Paul Griffiths, François Latraverse, Ron Mallon, Stephen Stich et Daniel Weinstock pour leurs aide et commentaires à différentes étapes de la rédaction de cet article. Cette étude a été rendue possible grâce au soutien du FCAR. 
Churchland 1988."Reduction and the Neurological Basis of Consciousness ». In Consciousness in Contemporary Science, sous la direction de A. Marcel et E. Biasach, Oxford : Oxford University Press, p. 273-304.

Clark, S. 1986. «Emotions : Rationality without Cognitivism ». Dialogue, XXV, p. 663-674.

Damasio, A.D. 1993. "Comprendre les fondements naturels des conventions sociales et de l'éthique, données neuronales ». In Fondements naturels de l'éthique, sous la direction de J.P. Changeux, Paris : Odile Jacob, p. 121-137.

Damasio, A., D. Tranel et H. Damasio. 1990. "Individuals with Sociopathic Behavior Caused by Frontal Damage Fail to Respond Autonomically to Social Stimuli ». Behavioral Brain Research, 41, p. 81-94.

D'Andrade, R. 1987. "A Folk Model of the Mind ". In Cultural Models In Language and Thought, sous la direction de D. Holland et N. Quinn, Cambridge, p.112-148.

De Sousa, R. 1980. "The Rationality of Emotions". In Explaning Emotions, sous la direction de A. O. Rorty, Berkeley : University of California Press, p. 127-151.

De Waal, F. 1997. Le bon singe : les bases naturelles de la morale. Paris: Bayard Éditions, 357 p.

Dumouchel, p. 1995. Émotions : essai sur le corps et le social. Paris : Synthélabo, coll. Les empêcheurs de tourner en rond.

Ekman, p. 1980a. «L'expression des émotions ». La recherche, 11, p. 14081415.

1980b. «Biological and Cultural Contributions to Body and Facial Movement in the Expression of Emotion ». In Explaning Emotions, sous la direction de A. O. Rorty, Berkeley : University of California Press, p. 73-101,

1984. "Expression and the Nature of Emotion ». In Approaches to Emotion, sous la direction de K. Scherer et p. Ekman, Hillsdale: Lawrence Erlbaum Associates, p. 319-344.

1992a. "An Argument for Basic Emotions ». Cognition and Emotion, 6, 169-200.

1992b. "Facial Expression of Emotion: New Findings, New Questions ». Psychological Science, 3, p. 34-38.

1992c. "Are there Basic Emotions ? ». Psychological Review, vol. 99, no. 3, p. 550-553.

1993. "Facial Expression and Emotion ». American Psychologist, April, Vol. 48, no. 4, p. 384-392.

1994. "All Emotions are Basic". In The Nature of Emotion: Fundamental Questions, sous la direction de p. Ekman et R. Davidson, Oxford : Oxford University Press, p. 15-19.

1998. "Afterword of Charles Darwin, The Expression of the Emotions in Man and Animals (édition définitive) ». Oxford : Oxford University Press. 
Ekman, p. et W. Friesen. 1971. "Constants Across Cultures in the Face and Emotion ". Journal of Personality and Social Psychology, 17, 2, p. 124129.

Ekman, P., R.W. Levenson et W. V. Friesen. 1983. "Automatic Nervous Activity Distinguishes Among Emotions ». Science, 221, p. 1208-1210.

Faucher, L. 1998. L'esprit fragmenté: psychologie évolutionniste et philosophie de la psychologie. Dissertation doctorale, UQAM. manuscrit. "What is it like to be funny and mind-myopic ».

Faucher, L. et S. Deragon. 1999. "Le tournant: déconstruction de la déconstruction ". Références, cadrages et ancrages, sous la direction de Nycole Paquin, Montréal : XYZ éditeurs.

Fernald, A. 1992. "Human Maternal Vocalizations to Infants as Biologically Relevant Signals : An Evolutionary Perspective ». In The Adapted Mind : Evolutionary Psychology and the Generation of Culture, sous la direction de J. Barkow, L. Cosmides et L. Tooby, Oxford : Oxford University Press, p. 391-428.

Fischer, A. et N. Frijda. 1992. "The Emotion Process as a Whole: A Response to Greenwood ». New Ideas In Psychology, vol. 10, no. 1, p.23-27.

Fodor, J. 1987. « Modules, Frames, Fridgeons, Sleeping Dogs, and the Music of the Spheres ". InThe Robot's Dilemma: The Frame Problem in Artificial Intelligence, sous la direction de Z. Pylyshyn, N.J. : Alex Publishing Corporation, p. 139-149.

Frank, R. 1988. Passions within Reason: The Strategic Role of Emotions. New York : W.W. Norton and Company.

Frank, R., T. Gilovich, et D. Regan. 1993. "The Evolution of One-Shot Cooperation: An Experiment ». Ethology and Sociobiology, 14, p. 247-256.

Frith, U. 1989. Autism : Explaining the Enigma, Oxford : Blackwell.

Gibbard, A. 1993. "Moralité et évolution humaine ». In Fondements naturels de l'éthique, sous la direction de J.-P. Changeux, Paris : Odile Jacob, p. 65-85.

Gould, J. et p. Marler. 1987. "Learning by Instinct ». Scientific American, p. 74-85.

Greenspan, p. 1981. "Emotions as Evaluations». Pacific Philosophical Quaterly, 62, p. 158-169.

Greenwood, J. 1992a. "The Social Constitution of Emotion ». New Ideas in Psychology, vol. 10, no. 1, p. 1-18.

1992b. "On the Kinds of Things that Are Emotions ». New Ideas in Psychology, vol. 10, no. 1, p. 29-33.

Griffiths, p. 1989. "The Degeneration of the Cognitive Theory of Emotions ». Philosophical Psychology, vol. 2, no. 3, p. 297-313. 1990. "Modularity and Psychoevolutionary Theory of Emotion ", Biology and Philosophy, 5 (2), p. 175-196. 
1994. "Cladistic Classification and Functional Explanation". Philosophy of Science, vol. 61, p. 206-227.

1997. What Emotions Really Are? Chicago: Chicago University Press.

Griffiths, p. et R. Gray. 1994. «Developmental Systems and Evolutionary Explanation ". The Journal of Philosophy, vol. 41, no. 6, p. 277-304.

Hamburg, D. 1968. " Emotions in the Perspective of Human Evolution ». In Perspective on Human Evolution, vol. 1, sous la direction de S. Washburn et p. C. Jay, New York : Holt, Riehart \& WInston, p. 246257.

Harré, R. 1986. "An Outline of the Social Constructionist Viewpoint ». In The Social Construction of Emotions, sous la direction de R. Harré, Oxford : Basil Blackwell, p. 2-14.

Harré, R. et R. Finlay-Jones. 1986. "Emotion Talk across Times ». In The

Social Construction of Emotions, sous la direction de R. Harré, Oxford : Basil Blackwell, p. 220-223.

Heelas, p. 1986. "Emotion Talk across Cultures". In The Social Construction of Emotions, sous la direction de R. Harré, Oxford : Basil Blackwell, p. 234-266.

Johnson-Laird 1988. The Computer and the Mind: An Introduction to Cognitive Science. Harvard : Harvard University Press.

Johnson-Laird, p. et K. Oatley. 1992. "Basic Emotions, Rationality, and Folk Psychology ». Cognition and Emotion, 6, no 3/4, p. 201-223.

Keltner, D. et B. Buswell. 1996. "Evidence for the Distinctness of Embarassment, Shame and Guilt : A Study of Recalled Antecedents and Facial Expressions of Emotion ". Cognition and Emotion, 10 : 2, p. 155-171.

Lazarus, R. 1984. « On the Primacy of Cognition ». American Psychologist, 39, p. 124-129.

1991. "Progress on a Cognitive-Motivational-Relational Theory of Emotion ". American Psychologist, 46, p. 819-834.

Ledoux, J. 1994a. "Cognitive-Emotional Interactions in the Brain ». In The

Nature of Emotion: Fundamental Questions, sous la direction de p. Ekman et R. Davidson, Oxford : Oxford University Press, p. 216223.

1994b. «Émotions, mémoire et cerveau ». Pour la science, no. 202, p. 50-57.

Levenson, R. 1994. "Human Emotions : A Functional View». In The Nature of Emotion: Fundamental Questions, sous la direction de p. Ekman et R. Davidson, Oxford : Oxford University Press, p. 123126.

Levy, R. 1984. "The Emotions in Comparative Perspective ». In Approaches to Emotion, sous la direction de K. Scherer et p. Ekman, Hillsdale : Lawrence Erlbaum Associates, p. 397-412. 
Lewis, M. 1993. «Self-Conscious Emotions : Embarrassment, Pride, Shame, and Guilt ». In Handbook of Emotions, sous la direction de M. Lewis et J. Haviland, New York : The Guilford Press, p. 563-573.

Lewis, M. et C. Saarni. 1987. "Culture and Emotion ». In The Socialization of Emotions, sous la direction de M. Lewis et C. Saarni, New York : Plenium Press, p. 1-17.

Loftus, E. et K. Ketcham 1994. The Myth of Repressed Memory: False Memories and Allegations of Sexual Abuse. NY : St. Matin's Press.

Lutz, C. 1982. "Introspection and Cultural Knowledge Systems ». Behavioral and Brain Sciences, 3, p. 439-440.

1986. "The Domain of Emotion Words on Ifaluk ». In The Social Construction of Emotions, sous la direction de R. Harré, Oxford : Basil Blackwell, p. 267-288.

Lutz, C. et G. M. White. 1986. «The Anthropology of Emotions ». Annual Review of Anthropology, 15, p. 405-436.

Maclean, p. 1980. "Sensory and Perceptive Factors in Emotional Functions of the Triune Brain ». In Explaning Emotions, sous la direction de A. O. Rorty, Berkeley : University of California Press, p. 9-36.

Marks, I. et R. Nesse 1994. "Fear and Fitness : An Evolutionary Analysis of Anxiety Disorders ». Ethology and Sociobiology, 15, p. 247-261.

Maynard-Smith, J. 1994. "L'évolution du comportement ". In Le comportement des animaux, Bibliothèque pour la science, Paris : Diffusion Belin, p. 82-97.

Mealey, L. 1995. "The Sociobiology of Sociopathy: An Integrated Evolutionary Model ». Behavioral and Brain Sciences, vol. 18, p. 523599.

Nesse, R. 1990. "Evolutionary Explanations of Emotions ». Human Nature, Vol. 1, no. 3, p. 261-289.

Oatley, K. 1993. "Social Construction of Emotions ". In Handbook of Emotions, sous la direction de M. Lewis et J. Haviland, New York : The Guilford Press, p. 341-352.

Oatley, K. et p. Johnson-Laird. 1987. «Towards a Cognitive Theory of Emotions ». Cognition and Emotion, vol.1, no. 1, p. 29-50.

Öhman, A. 1986. "Face the Beast and Fear the Face : Animal and Social Fears as Prototypes for Evolutionary Analyses of Emotion ". Psychophysiology, vol. 23, no. 2, p. 123-145.

Pankseep, J. 1989. "Les circuits des émotions ». Science et Vie, 168, p. 5867.

1992. "A Critical Role for «Affective Neuroscience » in Resolving What is Basic about Basic Emotions ». Psychological Review, vol. 99, no. 3, p. 554-560.

Plutchik, R. 1984. «Emotions : A General Psychoevolutionary Theory ». In Approaches to Emotion, sous la direction de K. Scherer et p. Ekman, Hillsdale : Lawrence Erlbaum Associates, p. 197-219. 
Rorty, A. 1980. «Introduction ». In Explaning Emotions, sous la direction de A. O. Rorty, Berkeley : University of California Press, p. 1-8.

Rozin, p. et A. Fallon. 1987. "A Perspective on Disgust ». Psychological Review, 94, p. 23-47.

Schacter, S. et J.E. Singer. 1962. "Cognitive, Social, and Physiological Determinants of Emotional State ». Psychological Review 69, p. 379399.

Scherer, K. 1993. « Neuroscience Projections to Current Debates in Emotion Psychology ». Cognition and Emotion, 7, no. 1, p. 1-41.

Searle, J. 1995. The Social Construction of Reality. New York : Free Press.

Segal, G. 1996. "The Modularity of Theory of Mind ». Theories of Theories of Mind, sous la direction de p. Carruthers et P.K. Smith, Cambridge : Cambridge University Press, p. 141-157.

Solomon, R. 1984. "Getting Angry: The Jamesian Theory of Emotion In Anthropology ". In Culture Theory: Essays on Mind, Self and Emotion, sous la direction de R. Shweder et R. LeVine, Cambridge : Cambridge University Press, p. 238-254.

1993. "The Philosophy of Emotions "In Handbook of Emotions, sous la direction de M. Lewis et J. Haviland, New York : The Guilford Press, p. 3-15.

Stearns, p. 1993. "History of Emotions: The Issue of Change ». In Handbook of Emotions, sous la direction de M. Lewis et J. Haviland, New York : The Guilford Press, p. 17-28.

Stich, S. 1996. Deconstructing the Mind. New York : Oxford University Press.

Tooby, J. 1987. "The Emergence of Evolutionary Psychology ». Emerging Synthesis In Science, p. 67-76.

Tooby, J. et L. Cosmides. 1990a. « The Past Explains the Present : Emotional Adaptations and the Structure of Ancestral Environments ». Ethology and Sociobiology, vol. $11: 375-424$.

Tulving, E. (1995). "Organization of Memory: Quo Vadis?». In The Cognitive Neurosciences, sous la direction de M. Gazzaniga, Cambridge : MIT Press, p. 839-847.

Wellman, H., p. Harris, M. Banerjee et A. Sinclair. 1995. "Early Understanding of Emotion: Evidence from Natural Language». Cognition and Emotion, 9, no. 2/3, p. 117-149.

White, G. 1993. "Emotions Inside Out : The Anthropology of Affect ». In Handbook of Emotions, sous la direction de M. Lewis et J. Haviland, New York : The Guilford Press, p. 29-39.

Zajonc, R. 1980. "Feeling and Thinking : Preferences Need no Inferences ». American Psychologist, 39, p. 151-175. 1984. "On the Primacy of Affect». American Psychologist, 39, p. 117-123. 